\title{
ANALYSIS OF INTERCONNECTED POWER SYSTEMS BY HYBRID CALCULATION
}

\author{
E. Ortjohann ${ }^{1}$, P. Wirasanti ${ }^{1}$, W. Sinsukthavorn ${ }^{1}$, S. Jaloudi ${ }^{1}$, D. Morton ${ }^{2}$ \\ ${ }^{1}$ Department of Electrical Engineering \\ South Westphalia University of Applied Sciences \\ Campus Soest - Lübecker Ring 2, 59494 Soest (Germany) \\ Phone: +0049 2921 378454, e-mail: ortjohann@fh-swf.de,paramet.por@gmail.com \\ ${ }^{2}$ The University of Bolton \\ Deane Road, Bolton, BL3 5AB, U.K.
}

\begin{abstract}
Distributed Energy Resources (DERs) are being added to the existing power supply systems to compensate for the growing electricity demand. This results in new power supply systems such as mini-grids and micro-grids being implemented into existing grids. In order to manage and optimize the operation of these new systems, power system analysis is utilized. However, the analysis model structure has to be recreated, once a new system joins the existing system. This results in an increased complexity of power system analysis, which calls for its simplification. The objective of this paper is to present a power system analysis method, which does not require restructuring of the analysis model in order to simplify the analysis of interconnected power systems. Hybrid calculation is applied in order to analyze the grid connecting points. This results the proposed analysis method can be performed by exchanging several parameters between the grid connecting points. The simulation results of the proposed method are compared with the general analysis in order to provide proof that the proposed method can be utilized as the general analysis method.
\end{abstract}

\section{Keywords}

Power system analysis, Interconnected power systems, Hybrid calculation, Dynamic-RMS model

\section{Introduction}

As the electricity demand is continuously increasing and the energy market expanding, DERs are quickly penetrating the market and being integrated into existing power systems. These form new power supply systems such as local distribution grids, mini-grids and microgrids. These can serve as electricity providers to power systems or to consumers from other generation resources. The cooperation between several new grids with the existing grid is necessary in order to manage, plan and optimize the power system, and avoid overloading of existing system components, when a new grid is added. Therefore, power system analysis is necessary to fulfill those requirements. Fig. 1 portrays a new grid joining an existing grid.

In general, the analysis of power systems uses a bus admittance matrix in order to define a system structure. If this bus admittance matrix is to be used when a new grid joins an existing grid, the consequence is a complex analysis, due to the complete recreation of the bus admittance matrix for the new system. This reduces the flexibility of the analysis and its ability to support the new grid. This is the reason why the analysis method has https://doi.org/10.24084/repqj09.239 to be adapted from the general analysis methods. This paper proposes a method to analyze the power system without the need to recreate the new bus admittance matrix, when a new grid joins an existing one.

In order to prove the proposed analysis method, the simulated power system needs a generation component, which provides a system frequency, terminal voltage and generates electrical power to support load demand. A diesel generator is one of the several generator components, which provides those requirements to the system, and is therefore used for the simulation case studies in this paper. However, the simulation of the full dynamic behavior of diesel generators is a timeconsuming process in complex systems. Thus, the dynamic-RMS model (see more details in [1]-[5]) is introduced, bringing with it further advantages. It reduces the computing time, which leads to a fast simulation of the complex power system, and uses less computational resources, while achieving the same behavior and almost similar results compared to a full scale dynamic model.

This paper is structured as follows: In chapter two, a general description of power system structure is given in order to define the bus admittance matrix. Chapter three clarifies the proposed interconnected power system analysis method. Moreover, the necessary exchange parameters between interconnected grids are introduced.

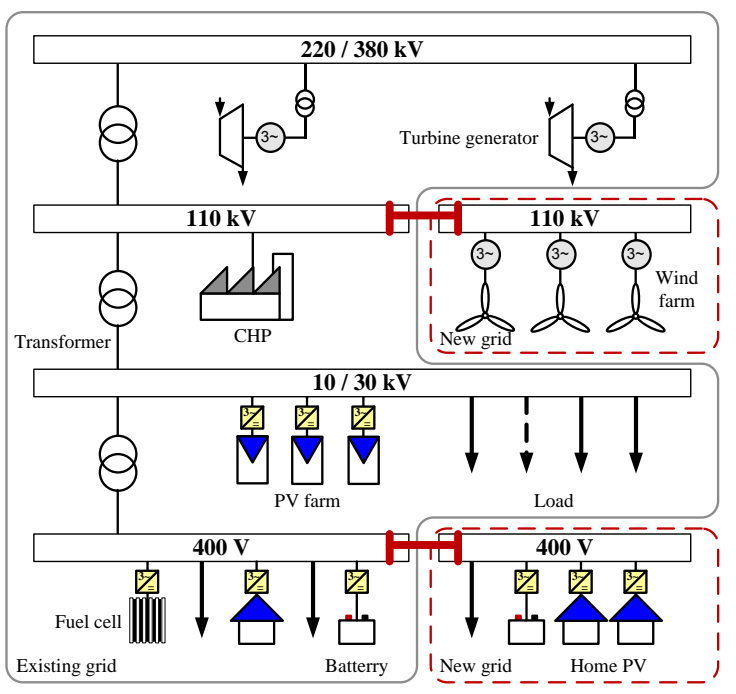

Fig.1. New grid joins the existing grid 
The dynamic-RMS model is introduced in chapter four. In chapter five, the simulation case studies are shown, the examined power system is simulated with the proposed analysis method and the recreation of bus admittance for the new system method is introduced. The power flow, the power balance, the system frequency, and the terminal voltage are mainly considered in the simulation. The simulation results are compared in order to show the difference between the two methods and to validate the proposed method.

\section{System definition}

A typical power system consists of electrical elements such as synchronous generators, loads, bus bars and transmission lines as shown in Fig. 2. The synchronous generator is represented as a voltage source connected in series with the impedance. Different types of this load can be identified as follows: active and reactive power load, active and reactive current load, resistance and reactance load and apparent power with power factor load. The transmission line between bus bars can be represented by using the $\pi$ - equivalent circuit. The grid network has to be portrayed in a single line diagram in order to represent it in a mathematical model in form of a bus admittance matrix. For example, the grid in Fig. 2 could be illustrated as in equation 1 .

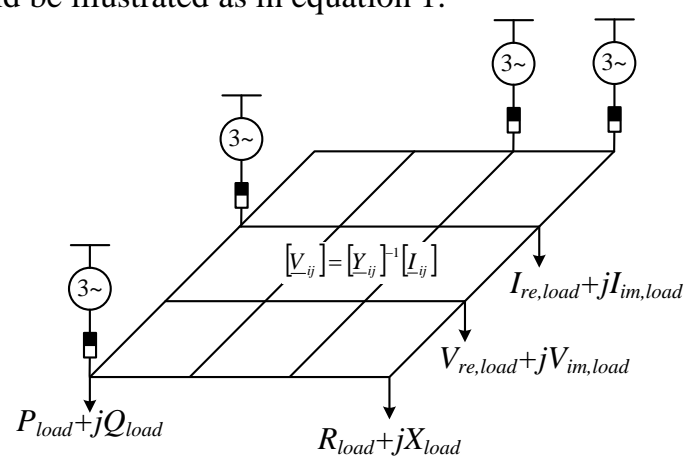

Fig. 2. A typical power system [1]

$$
\left[\begin{array}{c}
V_{1} \\
V_{2} \\
\vdots \\
V_{n}
\end{array}\right]=\left[\begin{array}{cccc}
Y_{11} & Y_{12} & \cdots & Y_{1 n} \\
Y_{21} & Y_{22} & \cdots & Y_{2 n} \\
\vdots & \vdots & \ddots & \vdots \\
Y_{n 1} & Y_{n 2} & \cdots & Y_{n n}
\end{array}\right]^{-1}\left[\begin{array}{c}
I_{1} \\
I_{2} \\
\vdots \\
I_{n}
\end{array}\right]
$$

Where $n$ is the number of buses in the power system, $V_{i}$ $(i=1,2,3, \ldots, n)$ is the complex vector of terminal voltages. $I_{i}(i=1,2,3, \ldots, n)$ is the complex vector of bus currents. $Y_{i i}$ $(i=1,2,3, \ldots, n)$ is the self-admittance of bus $i$, which is given at the diagonal elements. $Y_{i k}(i, k=1,2,3, \ldots, n)$ is the mutual admittance between buses $i$ and $k$.

In order to analyze the power system by applying the current method, all terminal voltages must be independent of the load and generator currents. Therefore, all load types have to be transformed into the active and reactive current load type. Similarly, the generator which is represented as a voltage source has to be transformed into a current source. (see more details about transform in [1]-[5])

\section{Analysis of interconnected power systems}

According to the analysis of interconnected power systems nowadays, new systems are being added into the existing systems to support increased electricity demand. The analysis method has to be adapted as well in order to support the changed system structure. In general, power system analysis is based on the bus admittance matrix, which defines a system structure. The bus admittance matrix has to be recreated in order to structure the new power system before analyzing it. Therefore, the analysis method which can be calculated separately on each grid is proposed.

As mentioned in chapter two, all bus currents have to be identified in order to conduct the power flow calculation using the applied current method. This means that the correct exchange current between the connected buses is an important parameter for the separation of the power flow analysis of interconnected grids. The exchange current is examined by following these three steps:

\section{A. Figure out the connected position of interconnected grids}

First of all, the structure of each grid and the connected position between interconnected grids has to be figured out. For example, introducing the interconnected grids as shown in Fig. 3.a. Fig. 3.a shows the interconnected grids structure, which has two grids. The connected position between the first grid and the second grid is the second bus and the fifth bus, respectively.

\section{B. Examine the exchange current by iteration with hybrid calculation}

After pointing out the connected position, the coupling area is defined. Fig. 3.b shows the grid structure with the coupling area, which refers to the connected buses, which are being added into each grid section; the fifth bus from the second grid is added to the grid area of the first grid and the second bus from the first grid is added to the grid area of the second grid. This coupling area provides the exchange parameters between the connected positions, which include the exchange current and the voltage of the connected buses. Since the voltage of the connected buses specifies the flow direction of the exchange current, this voltage has to be examined as the first step.

As mentioned, the analysis is done by the applied current method. However, the examination of the connected buses voltage cannot be transformed into the current form. Therefore, hybrid calculation is applied. Hybrid calculation is one of the calculation methods in [8], for which the input parameters can be the applied current and the applied voltage.

In order to calculate using the hybrid method, the bus admittance matrix has to be sorted by known parameters. Eq. 3 shows the sorting of the bus admittance matrix, where $N$ is an index of the bus for which the bus current is known and $\mathrm{L}$ is an index of the bus which the bus 
voltage is known. As a result, $\left[\mathrm{Y}_{\mathrm{NN}}\right]$ is the bus admittance matrix with known current buses. $\left[\mathrm{Y}_{\mathrm{LL}}\right]$ is the bus admittance matrix with known voltage buses. $\left[\mathrm{Y}_{\mathrm{NL}}\right]$ is the bus admittance matrix with known current buses and known voltage buses. [ $\left.\mathrm{Y}_{\mathrm{LN}}\right]$ is the transport of $\left[\mathrm{Y}_{\mathrm{NL}}\right]$. Then the hybrid matrix $[\mathrm{H}]$ can be calculated as illustrated in Eq.4.

$$
\begin{gathered}
{\left[Y_{\text {sorting }}\right]=\left[\begin{array}{cc}
{\left[Y_{N N}\right]} & {\left[Y_{N L}\right]} \\
{\left[Y_{L N}\right]} & {\left[Y_{L L}\right]}
\end{array}\right]} \\
{[H]=\left[\begin{array}{cc}
{\left[Y_{N N}\right]^{-1}} & -\left[Y_{N N}\right]^{-1}\left[Y_{N L}\right] \\
{\left[Y_{L N}\right]\left[Y_{N N}\right]^{-1}} & {\left[Y_{L L}\right]-\left[Y_{L N}\right]\left[Y_{N N}\right]^{-1}\left[Y_{N L}\right]}
\end{array}\right]}
\end{gathered}
$$

The calculation by the hybrid method is shown in Eq.5, in which the input vector consists of the known current vector $\left[\mathrm{I}_{\mathrm{N}}\right]$ and the known voltage vector $\left[\mathrm{U}_{\mathrm{L}}\right]$.

$$
\left[\begin{array}{c}
{\left[U_{N}\right]} \\
{\left[I_{L}\right]}
\end{array}\right]=[H] \times\left[\begin{array}{l}
{\left[I_{N}\right]} \\
{\left[U_{L}\right.}
\end{array}\right]
$$

This hybrid calculation examines the voltage and the exchange current of the connected bus, once again introducing the system in Fig. 3. From this grid structure, two hybrid matrices are created; one from the first grid area with the fifth bus added and one from the second grid area with the second bus added. This means that the buses from the grid areas are the known current buses and the added buses are the known voltage buses. Following, iteration processes with the hybrid calculation applied to find the connected buses voltage, are described:

- The first iteration process starts from the first grid area in order to find the terminal voltage of the second bus. The input vector is the bus current vector from the first grid area and the voltage of the fifth bus, which is set to the system nominal value.

- The second iteration process is performed on the second grid area to find the terminal voltage of the fifth bus. The input vector is the bus current vector from the second grid area and the voltage of the second bus, which is calculated from the first iteration.

The iteration process is repeated from the first step to the second step respectively until the connected buses voltage is convergent. At this point, the connected buses voltage is finalized. Then, the exchange current between the connected buses can be calculated by the voltage difference divided by the cable impedance.

\section{Analysis of the interconnected grids based on each grid}

After the exchange current is calculated, it will be added back to the corresponding bus. $I_{e x 25}$ is added back to the second bus and $I_{\text {ex52 }}$ is added back to the fifth bus, as shown in Fig. 3.c, where both exchange currents have the same value but in opposite directions.

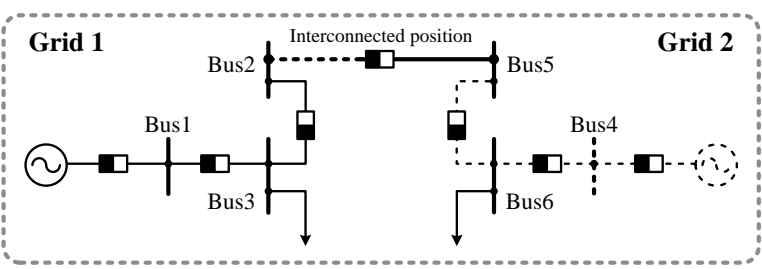

(a)
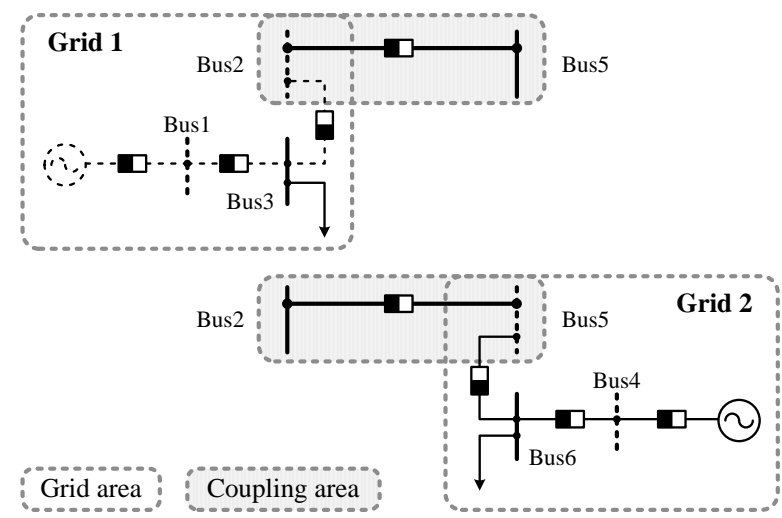

(b)
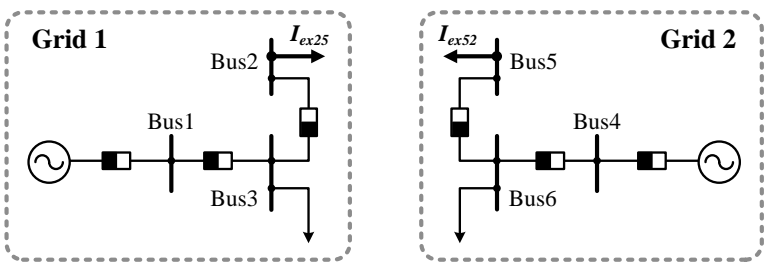

(c)

Fig. 3. (a) Interconnected grids

(b) Separated interconnected grids by coupling area

(c) Analysis of interconnected grids based on each grid

The exchange current represents the exchange power between the interconnected grids. In addition, Fig. 3.c portrays the interconnected grid power flow analysis for which two grids are calculated separately.

\section{Dynamic-RMS Model}

In order to perform a power system analysis of complex systems, the dynamic-RMS model in [1]-[5] is proposed to reduce the computing time but with the same behavior and result compared to full scale dynamic model. The model is performing based on an RMS value that can be used directly in power system analysis by applying the current method. Moreover, the model ignored the time constants of the diesel generator, which notably decreases the computational time. The diesel generator normally contains a diesel engine and a synchronous generator. The diesel engine is responsible for controlling the frequency and maintaining it to the nominal value. The synchronous generator, which is connected to the diesel engine, has to be able to keep the terminal voltages constant at any load variation. Fig. 4 shows a controlled diesel generator that implement control functions in order to fulfill the tasks of the diesel engine and the synchronous generator. In conventional power systems, the droop control function and/or the isochronous control function are often used for this purpose. 


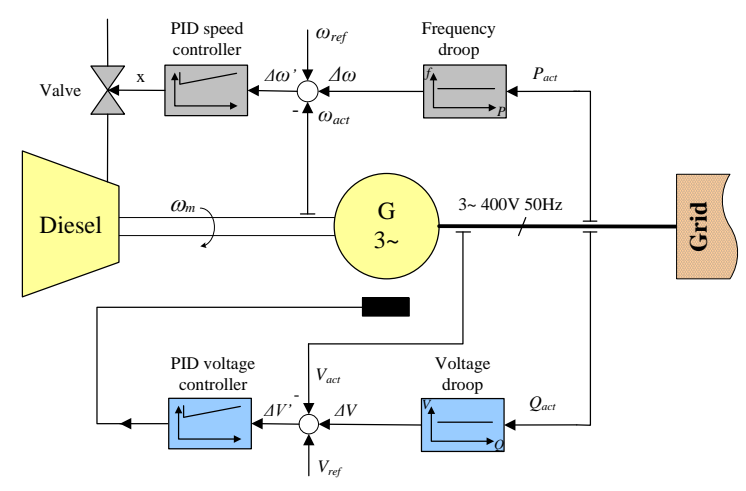

Fig. 4. Diesel generator with full droop control function [5]

In this paper, the full droop control function is used. According to Fig. 4, two droop control functions are implemented in the system; the frequency droop and the voltage droop. The frequency droop control is a linear factor, which is applied for controlling the diesel engine speed. The frequency droop factor is defined by the relationship between the angular reference speed and the active power of the synchronous generator. The droop controller is operated by measuring an actual active power and converted it into an angular speed offset. This means that the frequency will be maintained in an acceptable range of the nominal value. Likewise, the objective of the voltage droop controller is to maintain the terminal voltage close to the nominal value. The voltage droop factor is determined by the relationship between the voltage and the reactive power of the synchronous generator. Another function of droop controller is an electrical power sharing. This means that the droop controllers are applied when the model contains at least two generators. Otherwise, they are unnecessary.

The proof of this dynamic-RMS model is the comparative results of the terminal voltage of synchronous generator with the full scale dynamic fourth order model. A comparison between both results is shown in Fig. 5. The simulation shows that the dynamicRMS model has a slightly faster response than the full scale dynamic fourth order model. Although, the difference is very small, the dynamic-RMS model can be used to simulate a power system with an acceptable behavior compared to that of the full scale dynamic model.

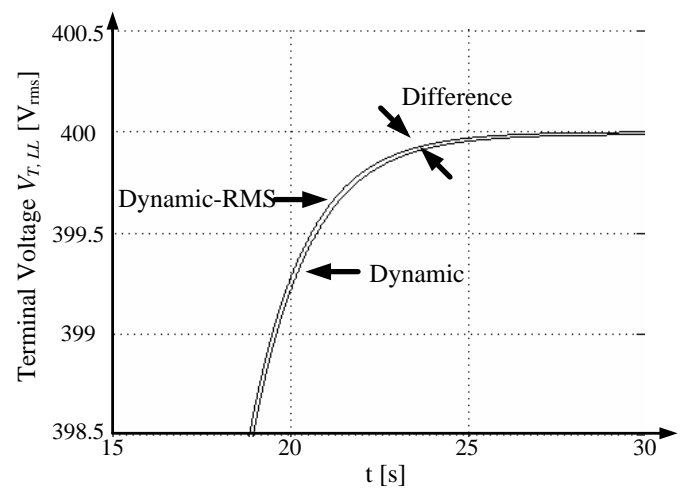

Fig. 5. Comparison between the simulation results of the dynamic-RMS model and the full dynamic model [1]

\section{Simulation case study}

To verify the proposed power system analysis method, a simulation case study as shown in Fig. 6 is examined. The system structure of the simulation case study has three isolated grids. The first grid has five buses with two generators, on the first and the second bus. The third bus is a load bus and the rest are connection buses to the second grid and the third grid. The second and the third grids are designed in the same structure; there is one generator on the first bus, load on the second bus, and connection buses on the third and the fourth. Dash lines show a grid section and the dot box shows a coupling point for each grid. There are three coupling points; the first grid on the fourth bus to the second grid on the third bus, the second grid on the fourth bus to the third grid on the fourth bus, and the first grid on the fifth bus to the third grid on the fourth bus. The proposed analysis method is examined in the first case study. The second case study is designed in order to compare the simulation results from the first case study, which all three isolated grids are defined as one grid structure.

The parameters for simulation case studies are described as following. All four generator parameters are identical; rated apparent power $(S)$ is $625 \mathrm{kVA}$, power factor is 0.8 , nominal voltage is $400 \mathrm{~V}_{\mathrm{L}-\mathrm{L}}$ and nominal frequency is 50 Hz. Each bus is connected with $100 \mathrm{~m}$ NAYY $4 \times 50 \mathrm{SE}$ cables; $\mathrm{R}_{1}=0.772 \Omega / \mathrm{km}$ and $\mathrm{X}_{1}=0.083 \Omega / \mathrm{km}$. The active and reactive power load is applied on the first grid at the third bus, the second grid at the second bus and the third grid at the second bus. At $\mathrm{t}=30 \mathrm{~s}$, a step load is applied on the third grid at the second bus. Table1 shows the value of active and reactive power load for each bus. The simulation results and comparison of both case studies are shown in Fig. 7 to Fig. 12 respectively; active power, reactive power, terminal voltage of generator bus and power system frequency.

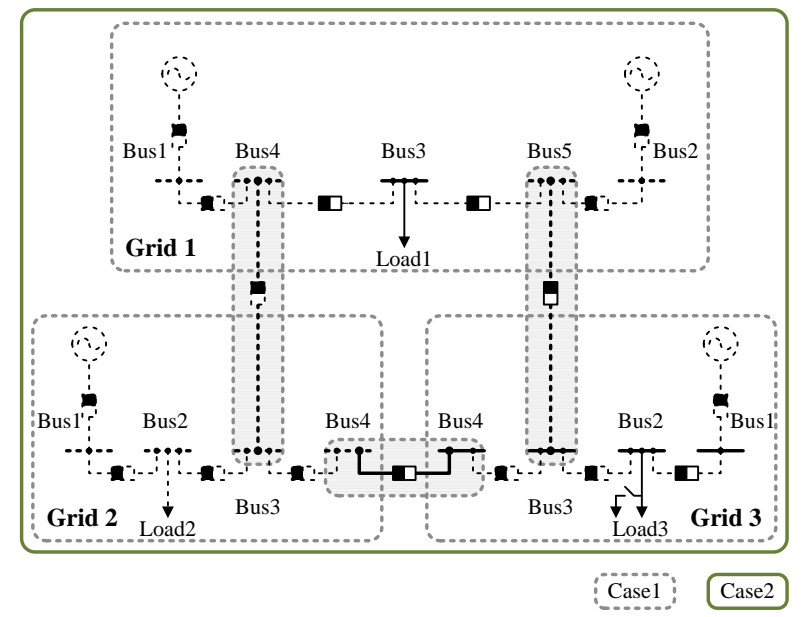

Fig. 6. Simulated power system, three grids with three interconnected positions

Table I. - Active- and reactive power load on simulated system

\begin{tabular}{|c|c|c|c|c|}
\cline { 2 - 5 } \multicolumn{1}{c|}{} & Load1 & Load2 & Load3 & Step load \\
\hline$P[\mathrm{~kW}]$ & 50 & 50 & 50 & 20 \\
\hline$Q[\mathrm{kvar}]$ & 25 & 25 & 25 & 10 \\
\hline
\end{tabular}


Fig. 7 shows the active power of all generators of both simulation case studies. Firstly, an active power flow balance is examined. The summation of the active power load demand is $150 \mathrm{~kW}$, this is supplied by all generators which is around $37.5 \mathrm{~kW}$ for each generator. After $\mathrm{t}=30 \mathrm{~s}$, the step load of $20 \mathrm{~kW}$ is added on the second bus of the third grid. This added load demand is supplied by all generators as well. The generator on the third grid is the most nearest to the added load, therefore it supplies more power than other generators.

According to the comparison in both simulation cases, the result shows the accuracy of the proposed analysis method. Fig. 8 shows the comparative result of the active power of the generator on the third grid. The results are the same; the difference cannot be observed, even in the zoom window. The dynamic behavior can be observed, it returns back to the steady state again after few seconds of the application of step load.

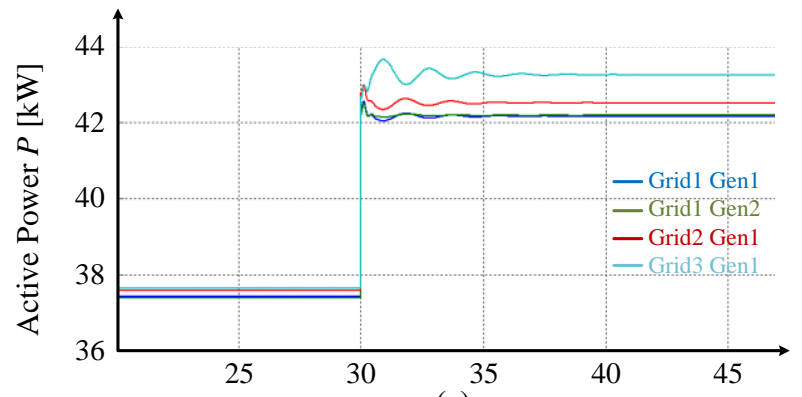

(a)

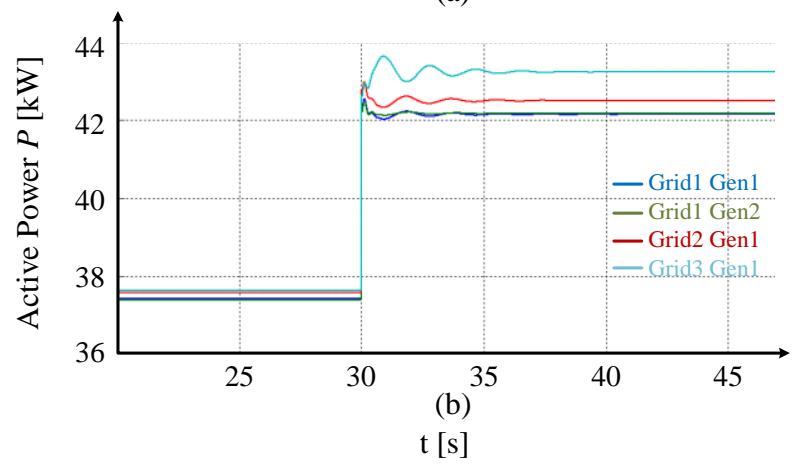

Fig. 7. (a) Active power of the generators, case 1

(b) Active power of the generators, case 2

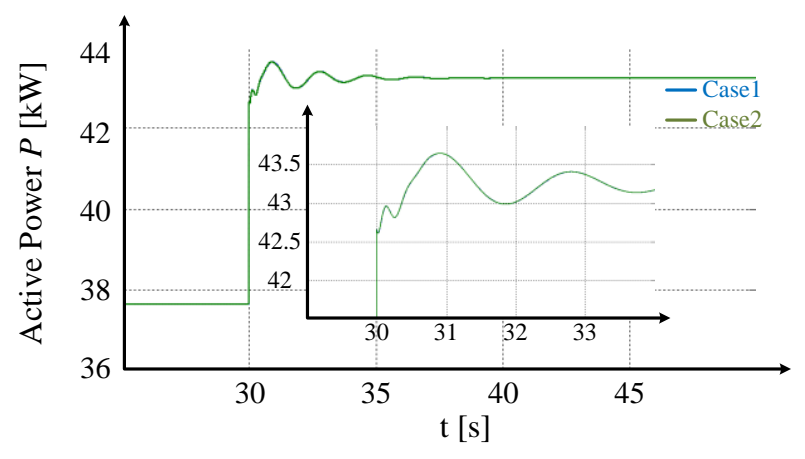

Fig. 8. Comparison between active powers of the generator on the third grid
The generated reactive powers of all generators in both case studies are shown in Fig. 9. The reactive power flow balance is inspected as well. Even though the sharing of reactive power is not the same for each generator, but the generated reactive powers are covered reactive power load for both period; before step load ( $Q=75 \mathrm{kvar}$ ) and after step load ( $Q=85$ kvar).

The comparative result of the generated reactive power of the generator on the third grid in both case studies is shown in Fig. 10. The comparison of reactive power results are the same even in dynamic phase during the step load. The zoom window is the proof of the comparison.

Normally, the terminal voltage is related to the reactive power. As shown in Fig. 10, there is no difference on the comparison of the reactive power. Consequently, the terminal voltage should be the same.

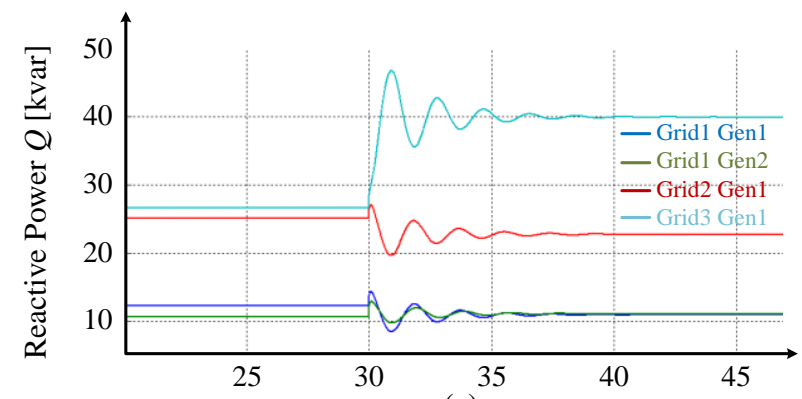

(a)

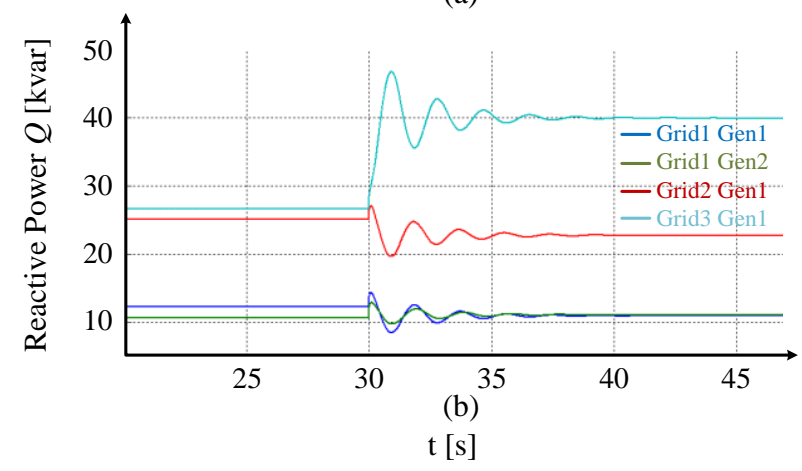

Fig. 9. (a) Reactive power of the generator, case 1

(b) Reactive power of the generator, case 2

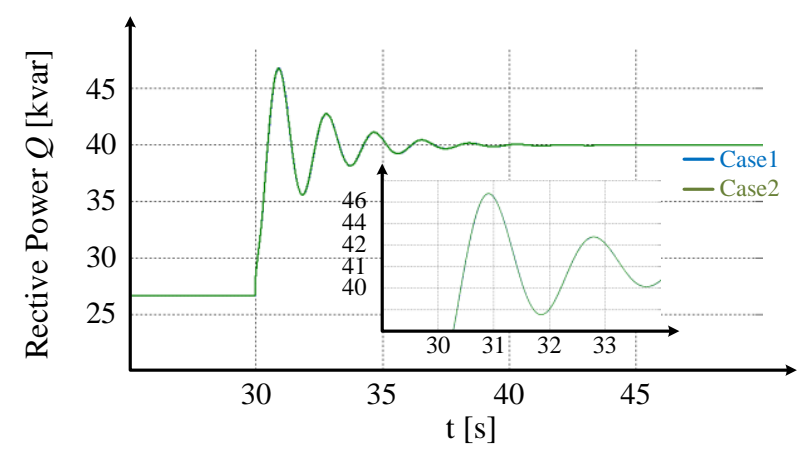

Fig. 10. Comparison between reactive powers of the generator on the third grid 
Fig. 11 shows the comparative result of the terminal voltage of the generator on the third grid. There is no difference of the comparative result of the terminal voltage of the generator bus on the third grid. The dynamic behavior is also correct. After the step load is added to the system, the terminal voltage is drop. And after few minutes, it returned back to the proper value.

The comparative result of the system frequency is shown in Fig. 12. Both simulation results are the same, which start with $50 \mathrm{~Hz}$ as nominal frequency and after the step load is added, the power system frequency is drop.

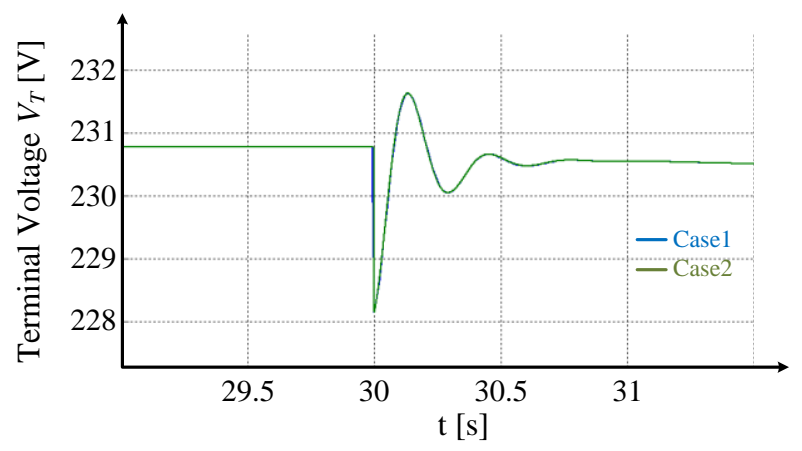

Fig. 11. Terminal voltage comparison of the generator on the third grid

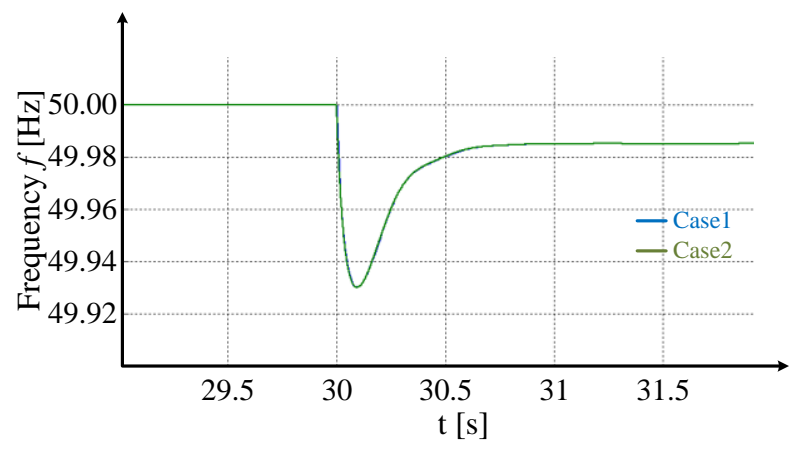

Fig. 12. System frequency comparison

\section{Conclusion}

This paper presents the analysis of interconnected power systems based on separated calculation by applying the current method for each grid. To perform the proposed analysis method, the exchanging current between gridconnected buses has to be examined by the iteration process using hybrid calculation, which this exchange current represent the exchange power. Afterward, it is added back into the corresponding connected position as the bus current. Then the applied current method analysis for each grid is used to calculate the power flow of the interconnected power systems. As a result, the proposed method does not require a change or recreation of the bus admittance matrix, in case of a new grid joining the existing grid.
The comparative result between the proposed analysis method and the general method provides proof for the suitability of the proposed method. As shown in the simulation results, the comparative result of active power, reactive power, terminal voltage and system frequency are the same.

The proposed analysis method can support the analysis of new grids connecting to the existing grids. In the DER system for example, new mini-grids and micro-grids are formed. These new grids can be analyzed parallel to the main grid without the need to change the main analysis model.

\section{Acknowledgements}

This research work is supported by the German "Federal Ministry of Education and Research" and administrated by the "Arbeitsgemeinschaft industrieller Forschungsvereinigungen "Otto von Guericke" e.V. (AiF)"'.

\section{References}

[1] E. Ortjohann, W. Sinsukthavorn, A. Mohd, N. Hamsic, M. Lingemann, D. Morton, "A Dynamic-RMS Modeling Method for Distributed Generation", IEEE Power Engineering Society 2009 General Meeting, Calgary, Alberta, Canada, (2009)

[2] E. Ortjohann, W. Sinsukthavorn, A. Mohd, N. Hamsic, A. Schmelter, D. Morton, "Dynamic-RMS Modeling of Distributed Electrical Power Supply Systems", IEEE PES Transmission and Distribution, Chicago, USA, (2008)

[3] E. Ortjohann, W. Sinsukthavorn, A. Mohd, N. Hamsic, A. Schmelter, D. Morton. "Modeling/Simulation of Power Distribution in Hybrid Power Systems Using Dynamic-RMS Technique", Portugal, (2007)

[4] E. Ortjohann, W. Sinsukthavorn, N. Hamsic, A. Schmelter, A. Mohd, D. Morton, "An Innovative Simulation Approach Using Dynamic-RMS model for Hybrid Isolated Mini-Grids", The Great Wall World Renewable Energy Forum and Exhibition 2006, Beijing, China, (2006)

[5] W. Sinsukthavorn, Master Thesis Active \& Reactive Power Dispatch Strategy in Isolated Mini-Grids with a Dynamic-RMS Model, University of Applied Sciences, Campus Soest, (2006), pp. 25-132.

[6] E. Ortjohann, O. Omari, Md. M. Rahman, D. Morton, Active \& Reactive Power Dispatch in Isolated miniGrids Fed by Decentralized Power Sources, 3rd International Conference on Electrical \& Computer Engineering, (2004)

[7] M. Hoppe, Diplomarbeit, Betriebführung elektrischer Netze mit dezentralen Stromerzeugern University of Applied Sciences, Campus Soest, (2009), pp. 85-105.

[8] K. Heuck, K. D. Dettmann, D. Schulz, Elecktrische Energieversorgung, Vieweg, (2007), pp. 365-389.

[9] A. J. Schwab, Elektroenergiesysteme, Springer Verlag, (2006), pp. 463-470.

[10] J. J. Grainger, W. D. Stevenson Jr., Power System Analysis, McGraw Hill Inc, (1994), pp. 238-280. 